\title{
Ice Lines in Circumbinary Protoplanetary Disks
}

\author{
Christian D. Clanton \\ Department of Astronomy, The Ohio State University, \\ 140 W. 18th Ave., Columbus, OH 43210, USA \\ email: clanton@astronomy.ohio-state.edu
}

\begin{abstract}
I find the location of the ice line in circumbinary disks heated by steady mass accretion and stellar irradiation, comparing the position with the minimum stable semimajor axis, interior to which planetary orbits are unstable. I show that there is a critical binary separation for which binaries with separations larger than this critical value have ice lines that lie interior to the boundary of stability. The critical separation for an equal-mass binary of $1 M_{\odot}$ stars is $\approx 1.04 \mathrm{AU}$, scaling weakly with mass accretion rate and Rosseland mean opacity of the disk. For a steady mass accretion rate of $\dot{M} \sim 10^{-8} M_{\odot} \mathrm{yr}^{-1}$ and a Rosseland mean opacity of $\kappa_{\mathrm{R}} \sim 1 \mathrm{~cm}^{2} \mathrm{~g}^{-1}$, I show that $\gtrsim 80 \%$ of all binary systems with component masses $M_{\star} \lesssim 2.0 M_{\odot}$ have ice lines interior to the boundary of stability. This suggests that rocky planets should not be common in these systems. Searching for planets around binaries with separations larger than the critical separation with Kepler or microlensing will provide a test of this prediction.
\end{abstract}

Keywords. binaries: general, planets and satellites: formation, stars: pre-main-sequence, planetary systems: protoplanetary disks

I adopt a simple disk model with the flared geometry described in Chiang \& Goldreich (1997), with a surface density distribution similar to the minimum mass Solar nebula (MMSN), $\Sigma=\Sigma_{0}(a / \mathrm{AU})^{-3 / 2}$, where $\Sigma_{0}=10^{3} \mathrm{~g} \mathrm{~cm}^{-2}$ (Weidenschilling 1977). Although I consider heating from both stellar irradiation and mass accretion, it turns out that the temperature of the disk midplane in regions of the disk where the ice line is expected to lie is dominated by mass accretion for binaries with component masses $M_{\star} \lesssim 2.0 M_{\odot}$. Fig. 1 plots the location of the ice line relative to the minimum stable semimajor axis as a function of binary separation for both equal-mass and unequal-mass binaries.

In the mass accretion-dominated regime, there is an analytic solution for the location of the ice line,

$$
a_{\mathrm{ice}}^{\mathrm{acc}} \approx 2.13 \mathrm{AU}\left(\frac{M_{\mathrm{tot}}}{M_{\odot}}\right)^{2 / 9}\left(\frac{\kappa_{\mathrm{R}}}{1 \mathrm{~cm}^{2} \mathrm{~g}^{-1}}\right)^{2 / 9}\left(\frac{\dot{M}}{10^{-8} M_{\odot} \mathrm{yr}^{-1}}\right)^{2 / 9}\left(\frac{T_{\text {ice }}}{160 \mathrm{~K}}\right)^{-8 / 9}
$$

where $M_{\text {tot }}$ is the total mass of the binary, $\kappa_{\mathrm{R}}$ is the Rosseland mean opacity of the disk, $\dot{M}$ is the mass accretion rate, and $T_{\text {ice }}$ is the temperature of ice condensation.

Comparing this with the minimum stable semimajor axis found by Holman \& Wiegert (1999), I find a critical binary separation, for which binaries with separations larger than this critical value have ice lines interior to the boundary of stability. For equal-mass binaries on circular orbits, this critical separation is $a_{\text {bin }}^{\text {crit }} \approx 0.89 \mathrm{AU}$, with the same scalings as (Eq. 0.1).

I compute the binary periods corresponding to the critical separations for equal-mass binaries and integrate over the observed binary period distribution of Raghavan et al. (2010) to estimate the fraction of binaries with ice lines interior to their inner disk edge. I find that ice lines lie interior to the minimum stable semimajor axis for $\gtrsim 80 \%$ of 


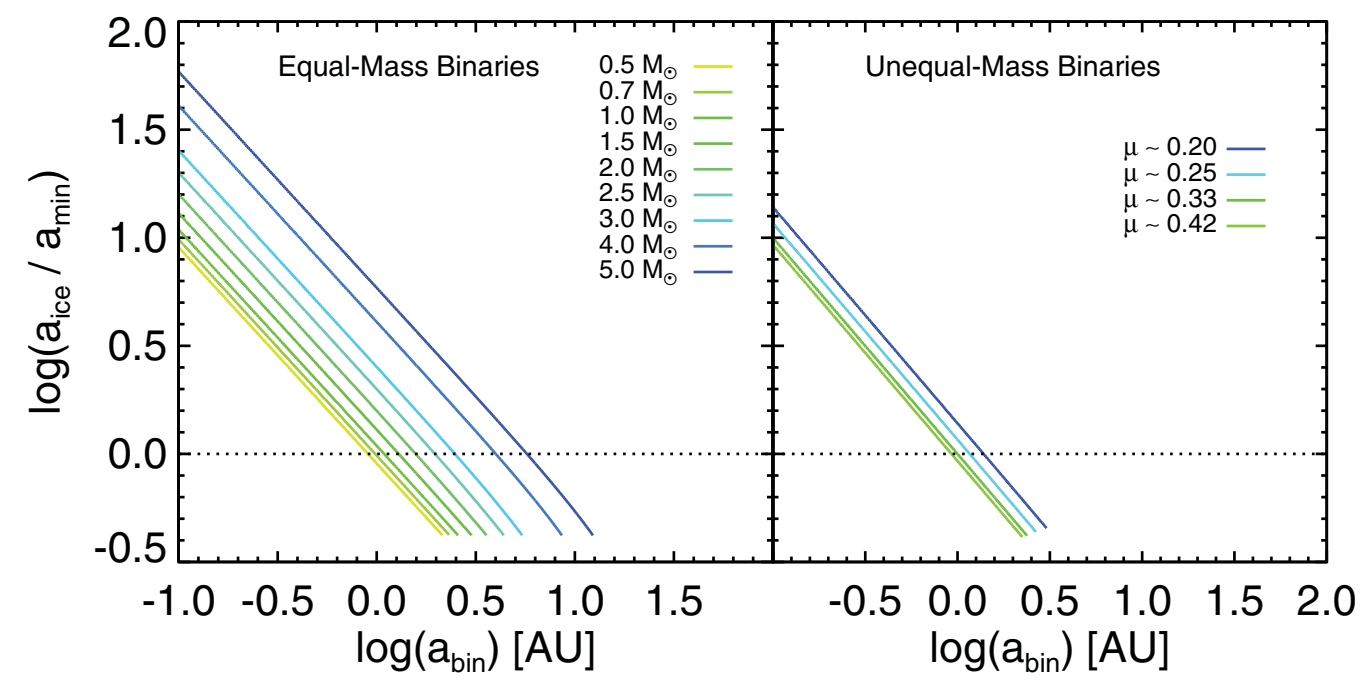

Figure 1. Ice line location relative to the minimum stable semimajor axis as a function of binary separation for both equal-mass (left) and unequal-mass binaries (right). Adapted from Clanton (2013).

all equal and unequal-mass binaries with components less massive than $M_{\star} \lesssim 2 M_{\odot}$. This suggests that rocky planets should not form in these systems. For more details, see Clanton (2013).

\section{References}

Chiang, E. \& Goldreich, P. 1997, ApJ 490, 368

Clanton, C. 2013, ApJ (Letters) 768, L15

Holman, M. \& Wiegert, P. 1999, AJ 117, 621

Raghavan, D., McAlister, H., Henry, T., Latham, D., Marcy, G., Mason, B., Gies, D., White, R., ten Brummelaar, T. 2010, ApJS 190, 1

Weidenschilling, S. 1977, Ap\&SS 51, 153 